\title{
ÜBER GEBIRGSBILDUNG UND TALSYSTEME IM NEPAL HIMALAYA
}

\author{
Toni Hagen
}

\section{E I N LE ITU NG}

Die ungewöhnliche Höhe des Himalayagebirges hat von jeher die Aufmerksamkeit der Forscher auf sich gezogen, und zahlreich sind die Versuche, dieses Phänomen zu deuten. Als besondere Eigenheit des Himalayas war schon lange bekannt, daß die Hauptkette mit den Achttausendern nicht gleichzeitig auch Hauptwasserscheide zwischen Tsangpo (Brahmaputra) und Ganges ist, sondern daß diese Scheide meist nördlich der Hauptkette liegt, und daß viele Flüsse die Hauptkette in gewaltigen Schluchten von Norden nach Süden durchbrechen.

In Nepal durchbrechen die folgenden großen Flüsse die Hauptkette des Himalaya: Karnali, Bheri, Kali Gandaki, Marsyandi, Buri Gandaki, Trisuli, Sun Kosi und Arun.

Diese Flüsse teilen den Himalaya in einzelne Gebirgsgruppen auf, von Westen nach Osten: Saipal Himal, Kanjiroba Himal, Dhaulagiri $8171 \mathrm{~m}$, Annapurna $8078 \mathrm{~m}$, Gurkha Himal (Manaslu $8128 \mathrm{~m}$ ), Ganesh Himal und Langtang Himal.

Die ausführlichsten Arbeiten, die bisher das eigentümliche Phänomen des Durchbruches der Flußsysteme zu ergründen suchten, stammen von L. R. WAGER, von P. G. BurRard und von H. H. Hayden. Diese Arbeit wurde 1943 durch Burrard und Heron revidiert.

Zwei Theorien zur Erklärung des Durchbrechens der Himalayaflüsse haben sich bisher herauskristallisiert. Nach der einen bestand das Hauptgewässernetz schon vor der Auftürmung des Himalayas zu seiner heutigen Höhe. Die weit im Norden entspringenden Flüsse hätten demnach gleichzeitig mit der Hebung die sich auftürmenden Gebirge durchbrochen. Die zweite Theorie begründet die Entstehung der Taldurchbrüche aus Anzapfungen tibetischer Flüsse von Süden her. WAGER hat speziell das System des Arun Flusses untersucht, der die Hauptkette des Himalayas zwischen Everest und Kangchendzönga durchbricht. Er stellte am Arun fest, daß sein Längsprofil im Unterlauf (in Höhen bis ca. $1200 \mathrm{~m}$ ) eine flache Kurve, typisch für einen reifen Fluß zeichnet, während er im Durchbruch einen bedeutenden Gefällsknick aufweist, um nördlich der Hauptkette wiederum flach zu verlaufen. WAGER hält sich an die Theorie vom vorherbestehenden Gewässernetz und nachheriger Hebung des Himalayas.

Es war zu WaGers Zeiten schon erwiesen, daß die letzte Hebung des Himalayas geologisch sehr spät, nämlich ins Pliozän zu datieren ist, und daß sie heute noch anhält. C. S. MidDlemiss (1923) zeigte schon, daß die rezenten Karewa Formationen über die Pir Parisad Range mit Fallbeträgen bis zu $50^{\circ}$ gelagert sind!

Seit langem war ferner bekannt, daß die heutige Höhe der Himalaya-Hauptkette das Resultat von horizontalen Schüben mit Aufpressen sowie von direkter Hebung ist. Der ersteren wurde jedoch nur bescheidene Bedeutung zugemessen.

Odell (1925) führte aus, daß das Gebiet des obern Aruntales einen quartären Eisschild besessen habe und daß nach dessen Abschmelzen jenes Gebiet sich infolge isostatischen Ausgleiches gehoben hätte. WAGER (1937) verneinte das Bestehen eines ehemaligen Eisschildes, und auch der Verfasser fand auf seinen ausgedehnten Expeditionen nur sehr geringe eiszeitliche Moränen, die nicht von einem großen Eisschild, sondern nur von lokalen, ehemaligen Talgletschern herrühren können.

Nach WaGer entspricht die Überhöhung des Himalayas (rund $3000 \mathrm{~m}$ ) über das tibetische Plateau von $4000 \mathrm{~m}$ dem nach dem Ausräumen der großen Täler wieder hergestellten isostatischen Gleichgewicht. In seiner Publikation (Everest 1933) statuierte WAGER: „Wenn man im Sikkim Himalaya das Gebirge nivellieren und cie Täler mit dem anfallenden Material ausfüllen könnte, würde daraus ein Plateau entstehen, dessen Oberfläche sanft gegen die Gangesebene hinunter fällt, und im Gebiet der heutigen Hauptkette hätte das Plateau eine Höhe von rund $4600 \mathrm{~m}$, was ganz wenig niedriger ist als der unmittelbar nördlich davon gelegene Teil des tibetischen Plateaus. WAGER nimmt hier den ehemaligen Rand des tibetischen Plateaus an, mit sanftem S-Fallen. Die zum Ganges System gehörigen Flüsse hätten ihren Ursprung dort gehabt, dann aber zufolge stärkerer Erosion von Süden (mehr Gefälle und Niederschlag) sich nach rückwärts (Norden) eingeschnitten. Um den durch die Erosion verursachten Massendefekt auszugleichen, sei der Rand des tibetischen Plateaus direkt gehoben worden. $\mathrm{Er}$ unterscheidet also 2 Phasen im Bau des Himalayas: 1. Hebung des tibetischen Plateaus infolge horizontaler Schübe; 2. vertikale Hebung des ehemaligen südlichen Plateaurandes. Massendefekt infolge Abschmelzen des quartären Eisschildes und Erosion und Wiederherstellung der Isostasie. Daraus resultierten die Überhöhungen von $3000 \mathrm{~m}$ der Himalaya Hauptkette über dss tibetische Plateau und damit die abnormen Gesamthöhen von $7000-8000 \mathrm{~m}$.

Bemerkenswert sind auch die Beobachtungen von DyHRENFurTh, wonach sich die Achttausender durch einen auffallenden, wie von vertikalen Verwerfungen herrührenden Steilwandgürtel auszeichnen. 


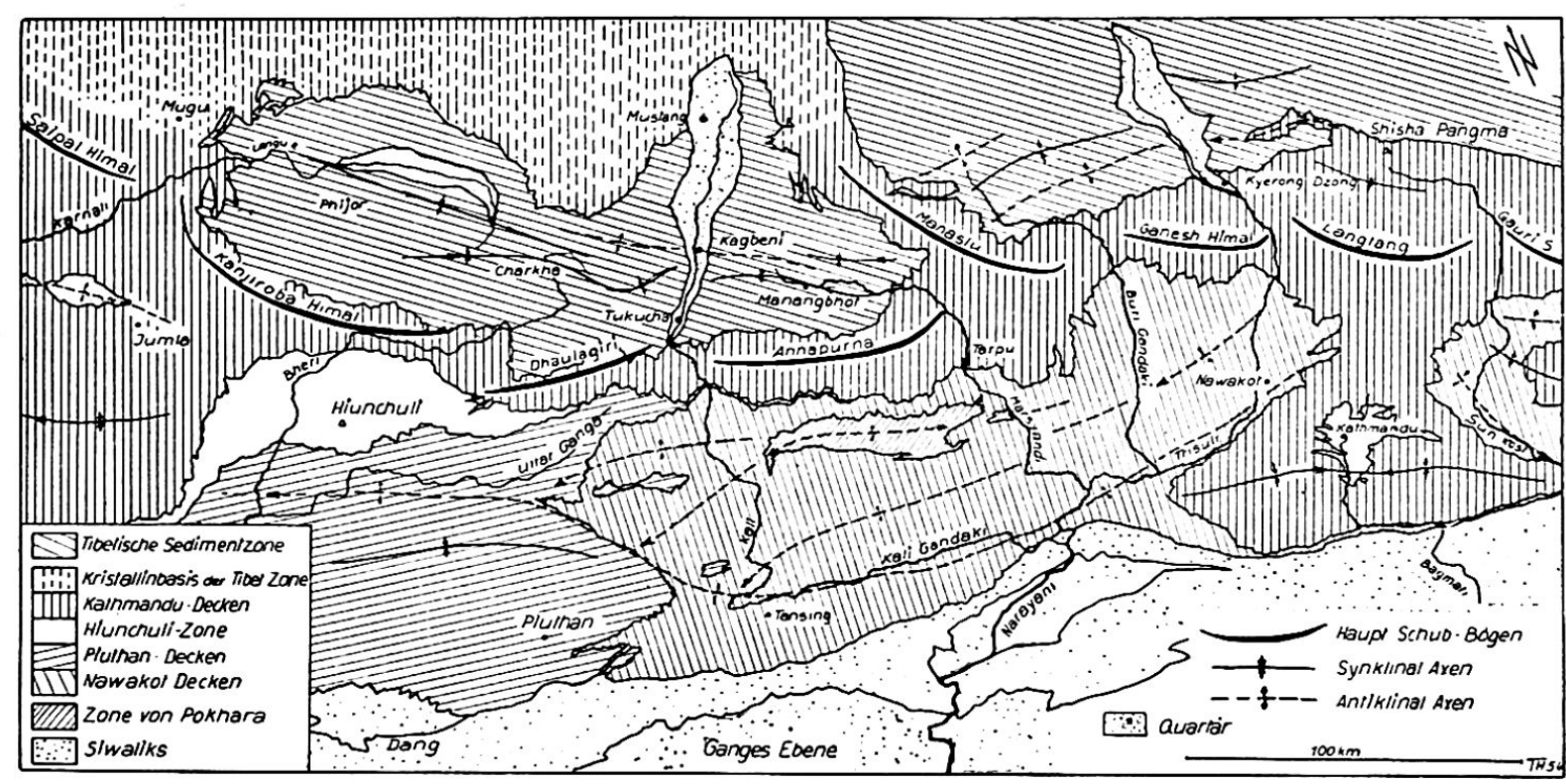

Fig. 1. Tektonische Skizze von Zentral-Nepal

DIE EIGENEN BEOBACHTUNGEN

Dem Verfasser war es vergönnt, in 3-jähriger Feldarbeit (wobei ca. $6000 \mathrm{~km} \mathrm{zu}$ Fuß zurückgelegt worden sind), einen umfassenden Einblick in die Geographie und Geologie des Nepal Himalayas zu gewinnen. Dabei wurde mehr und mehr klar, daß Topographie und Gewässernetz weitgehend von der Tektonik bestimmt sind. $\mathrm{Zu}$ nächst seien daher die tektonischen Grundzüge von Nepal kurz skizziert.

Von oben nach unten konnten unterschieden werden: 1. Kathmandu-Decken (4-5), 2. Zone des Himalchuli (4 Schuppen), 3. Piuthan-Decken (5 Decken), 4. Nawakot-Decken (6 Decken), sowie 5. Zone von Pokhara.

Diese Gliederung ist natürlich nur eine erste übersichtsmäßige (die geologische Originalkartierung erfolgte im Maßstab 1:250000). Die Nawakot-Decken sind in Zentral-Nepal am besten entwickelt (vergl. Fig. 1) zwischen Bari Gad Fluß und 'Trisuli, mit Überschiebungsbeträgen bis $75 \mathrm{~km}$. Die Piuthan-Decken und die Himalchuli-Zone sind nur in West-Nepal vorhanden. Die Zone von Pokhara ist das tektonisch tiefste Element und dürfte auf Grund spezieller N-S gerichteter Strukturen event. dem Parauthochthon zugeschrieben werden. Die Gesteinsinhalte der Deckengruppen 2, 3, 4 und 5 sind ähnlich, und umfassen Formationen von Karbon bis Unter-Jura. Die Kathmandu-Decken bilden inbezug auf das Relief das Hauptelement: Ihre überschobenen Teile finden sich in den Halbklippen von Kathmandu, dann in den großen Massen des Thakurjigebirges südlich Jumla, und in vorderhand noch nicht näher untersuchten Gebieten in Ost-Nepal (HAGEN 1952). Die Kathmandu-Decken bestehen aus Formationen, welche Stufen vom Silur an abwärts umfassen. Sie zeichnen sich aus durch große Intrusionen von Graniten und mannigfache Metamorphose-Erscheinungen in den Nebengesteinen (Hagen 1952). Die Wurzelzone der Kathmandu-Decken ist identisch mit der Hauptkette des Himalayas.

Nördlich an die Wurzelzone der Kathmandu-Decken schließt sich die Tibetische Zone an mit Formationen, die sich vom Kristallin der Kathmandu-Decke V über die silurischen und devonischen Kalke lückenlos bis zur oberen Kreide erstrekken. Der Südteil des tibetischen Plateaus ist gegen die Wurzelzone der KathmanduDecken zunehmend verfaltet und verschuppt. Decken dagegen treten hier nicht mehr auf. Diese kompliziert gebaute Zone entlang dem N-Rand der Kathmandu-DeckenWurzelzone sei die Randschuppenzone genannt.

Nördlich der Ketten von Dhaulagiri und Annapurna besteht die genannte Randschuppenzone aus einem komplizierten Synklinorium, der Synklinalzone von Phijor 
- Charkha - Kagbeni und Manangbhot. Nördlich dieser Synklinalzone, im Gebiet von Mustang, erscheint wiederum die Kristallinbasis. Westlich Phijor, bei Mugu und östlich von Manangbhot, bei Larkya ist sie durch axialen Anstieg direkt sichtbar mit dem Kristallin der Kathmandu-Decke V in der Wurzelzone verbunden.

Aus dem Granitstock von Mustang entwickelt sich in Südost Richtung die Wurzelzone der Kathmandu-Decke V im Manaslu, welche diskordant zu den Strukturen in der Annapurna streicht (Fig. 1).

Der Schubbogen des Ganesh Himal ist gegenüber demjenigen des Manaslu ca. $10 \mathrm{~km}$ nach Norden versetzt und auch sein Streichen dreht nach Ost-Süd-Ost ab. Östlich an den Ganesh-Bogen schließt sich der Langtang-Bogen an. Dieser ist der Kristallinaufwölbung der Shisha Pangma (Gosainthan), $8013 \mathrm{~m}$ vorgelagert. Östlich der Shisha Pangma weisen die Strukturen und damit das Streichen der Wurzeln der Kathmandu-Decken zunächst wieder Süd-Ost-Richtung auf. Das Kristallin-Massiv der Shisha Pangma gehört nicht zu den Wurzeln der Kathmandu-Decken, sondern es liegt in der axialen Kulmination einer tibetischen Kristallin-Struktur. In tektonischer Hinsicht stellt es einen festen Pivot dar, um welchen die Wurzeln der KathmanduDecken beidseits nach Süden gepreßt wurden.

Die ganze Kette des Himalayas teilt sich nun, entsprechend der topographischen Aufgliederung, in einzelne Schubbögen auf, deren Wirkung in den Streichrichtungen bis gegen die Siwaliketten (Molasse am Rand der Gangesebene) hinunter zu sehen ist. Chronologisch sind folgende Schubbögen festzustellen:

1. Annapurna-, 2. Dhaulagiri-, 3. Kanjiroba-, 4. Saipal-, 5. Manaslu-, 6. Ganesh-, 7. Langtang-, 8. Gaurisankar-, Everest-Bogen.

Der Annapurna-, DhaulagiriBogen ist der zentrale Ausgangspunkt der Überschiebung der Kathmandu - Decken. Um diese Zone haben sich alle spätern Phasen der Aufrichtung der Wurzeln der Kathmandu-Decken gruppiert, z. T. diskordant. Auch alle Durchbruchsflüsse haben ihr Ursprungsgebiet irgendwie nördlich des Annapurna-, DhaulagiriBogens und divergieren aus diesem Raume heraus (Fig. 1). Merkwürdig genug, daß gerade dieser sonst einheitlich scheinende Bogen vom Kali Gandaki scheinbar gerade durchbrochen wird, während sonst alle Flüsse, welche nördlich der Hauptkette entspringen, ihren Weg nach Süden zwischen den einzelnen tektonischen Bogen oder Segmenten suchen, nämlich :

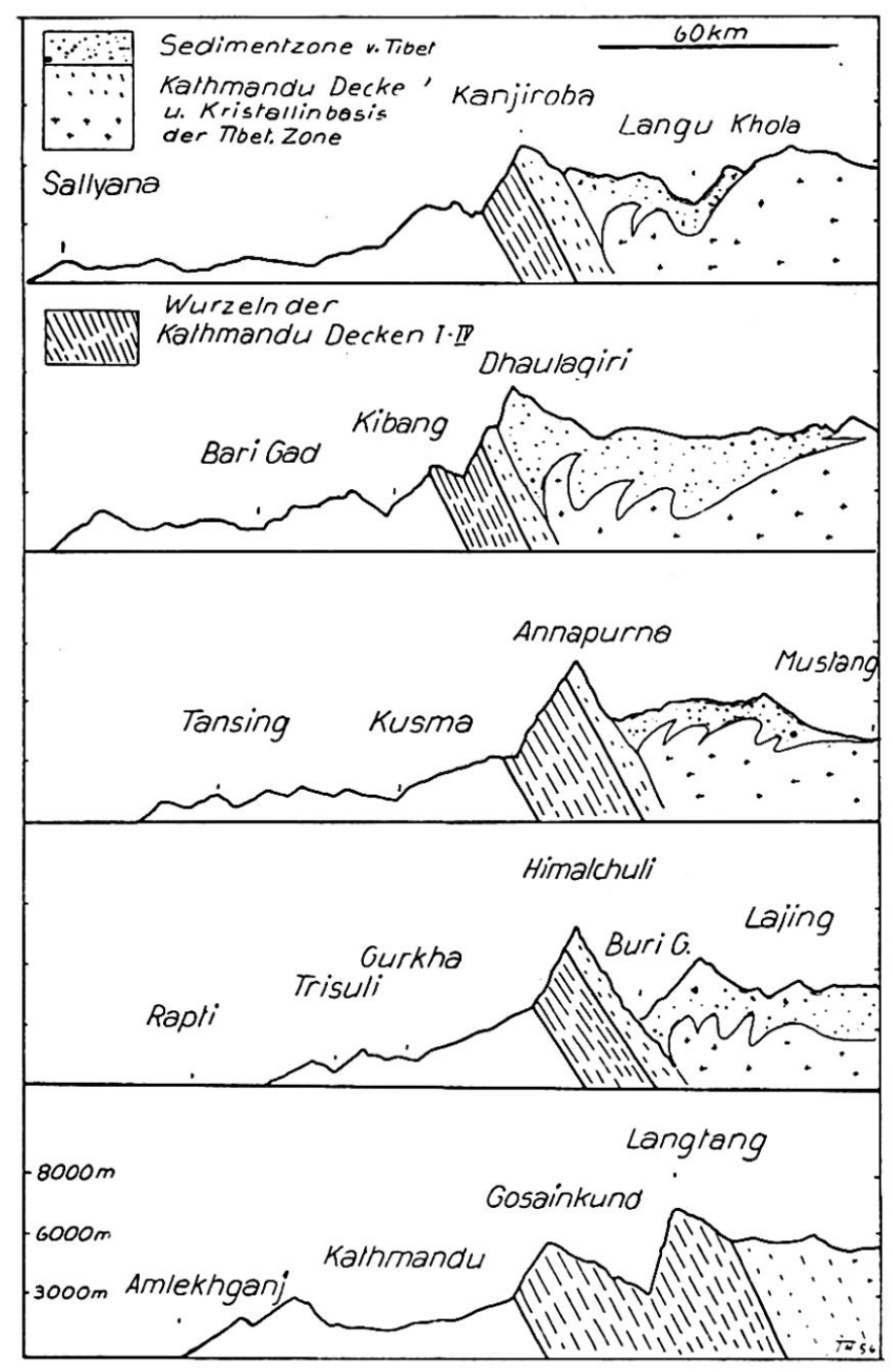

Fig. 2. Querprofile durch den ${ }_{a}^{-N e p a l ~ H i m a l a y a, ~} 5$ fach iiberhöht. Die höchsten Erhebungen in der Hauptkette des Himalaya liegen in den Wurzelzonen der Kathmandu-Decken $\mathrm{I}-\mathrm{V}$ 


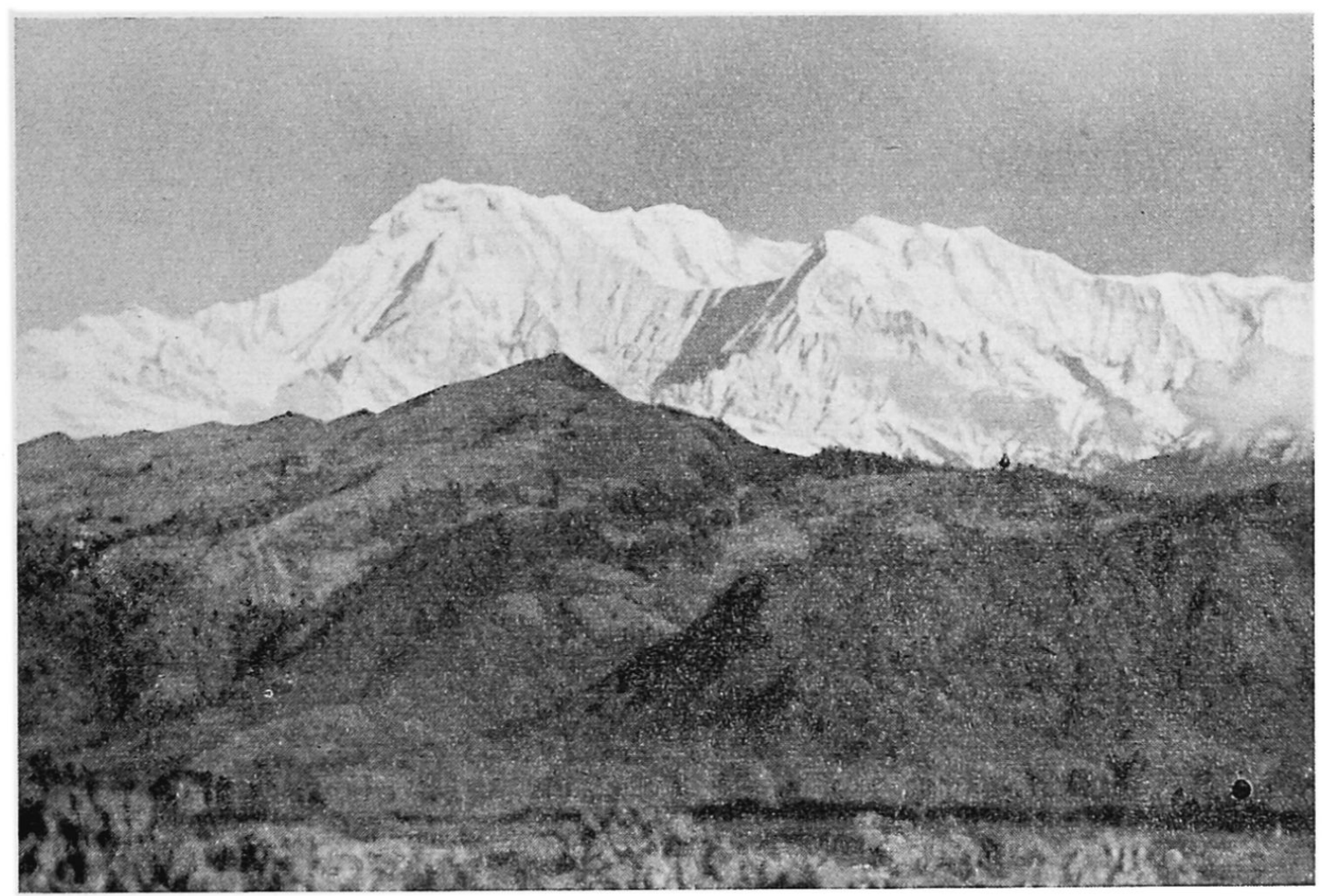

Fig. 3. Die Annapurna-Kette von der Ebene von Pokhara aus (von Süden). Die Ebene im $25-30 \mathrm{~km}$, und praktisch ohne Vorgebirge erhebt sich die Kristallinmasse aus dem Tiefland bis gipfel, $8078 \mathrm{~m}$ (vorwiegend Granite), Machhapuchhare (= Fischschwanz, vorwiegend Granite im

- der Langu-Karnali Fluß mit Einzugsgebiet nördlich der Kanjiroba- und Dhaulagiri Kette durchbricht die Hauptkette zwischen Saipal und Kanjiroba Bogen,

- der Bheri Fluß mit Einzugsgebiet an der Nordflanke der Dhaulagiri Kette durchbricht die Hauptkette zwischen Kanjiroba- und Dhaulagiri Bogen,

- der Kali Gandaki durchbricht die Hauptkette zwischen Dhaulagiri- und Annapurna Bogen,

- der Marsyandi entwässert die Nordseite der Annapurna und durchbricht den Hauptkamm zwischen dieser und dem Manaslu Bogen,

- der Buri Gandaki entwässert die Nordflanke der Manaslu Gruppe und zwängt sich zwischen dieser und dem Ganesh Bogen nach Süden,

- der Trisuli Fluß nimmt seinen Ursprung sehr weit im Norden und durchbricht die Hauptkette zwischen dem Ganesh- und dem Langtang Bogen, und endlich

- der Sun Kosi sucht seinen Weg nach Süden zwischen Langtang- und Gauri Sankar Bogen. Trisuli Fluß und Sun Kosi umfließen in symmetrischer Anordnung die Halbklippe der Kathmandu-Decken.

Aber auch der Kali Gandaki hat seine besondere tektonische Geschichte: Bei wiederholter Begehung des Uttar Ganga und des Gurja Flusses südlich der Dhaulagiri Gruppe fiel immer wieder auf, daß der Oberlauf des letzteren durch das bedeutend tiefere und wilde Tal des Darkhola quer abgeschnitten wird. Auch fehlt heute ein Fluß, der für die Schaffung des weiten Talkessels von Dhorpatan verantwortlich gemacht werden könnte. Eindrucksvoll gestaltet sich die Sicht vom Lumsum $\mathrm{Pa}$ am obern Ende des weiten Tales des Uttar Ganga $(3100 \mathrm{~m})$ : Nach Westen erblickt man den weiten, alpin anmutenden, geraden Tallauf des Uttar Ganga, der ganz minimes Gefäll aufweist. Nach Nordosten geht es an die $1000 \mathrm{~m}$ hinunter ins Tal von Lumsum und in das Mayangdital. Ein «Malojapaß»größten Ausmaßes! Noch weiter nach Osten und Ost-Nord-Ost schweift der Blick in die Senke des Kali-Tales zwischen Annapurna und Dhaulagiri. Besonders auch vom Flugzeug aus drängt sich die Erkenntnis geradezu auf, daß das westwärts verlaufende Tal des Uttar Ganga die ursprüngliche Fortsetzung des Kalitales ist. Ein westlicher Seitenfluß des Mayangdi Kolas hat den Ur Kali im Gebiet des heutigen Dar Kholas seitlich angezapft. Aber 


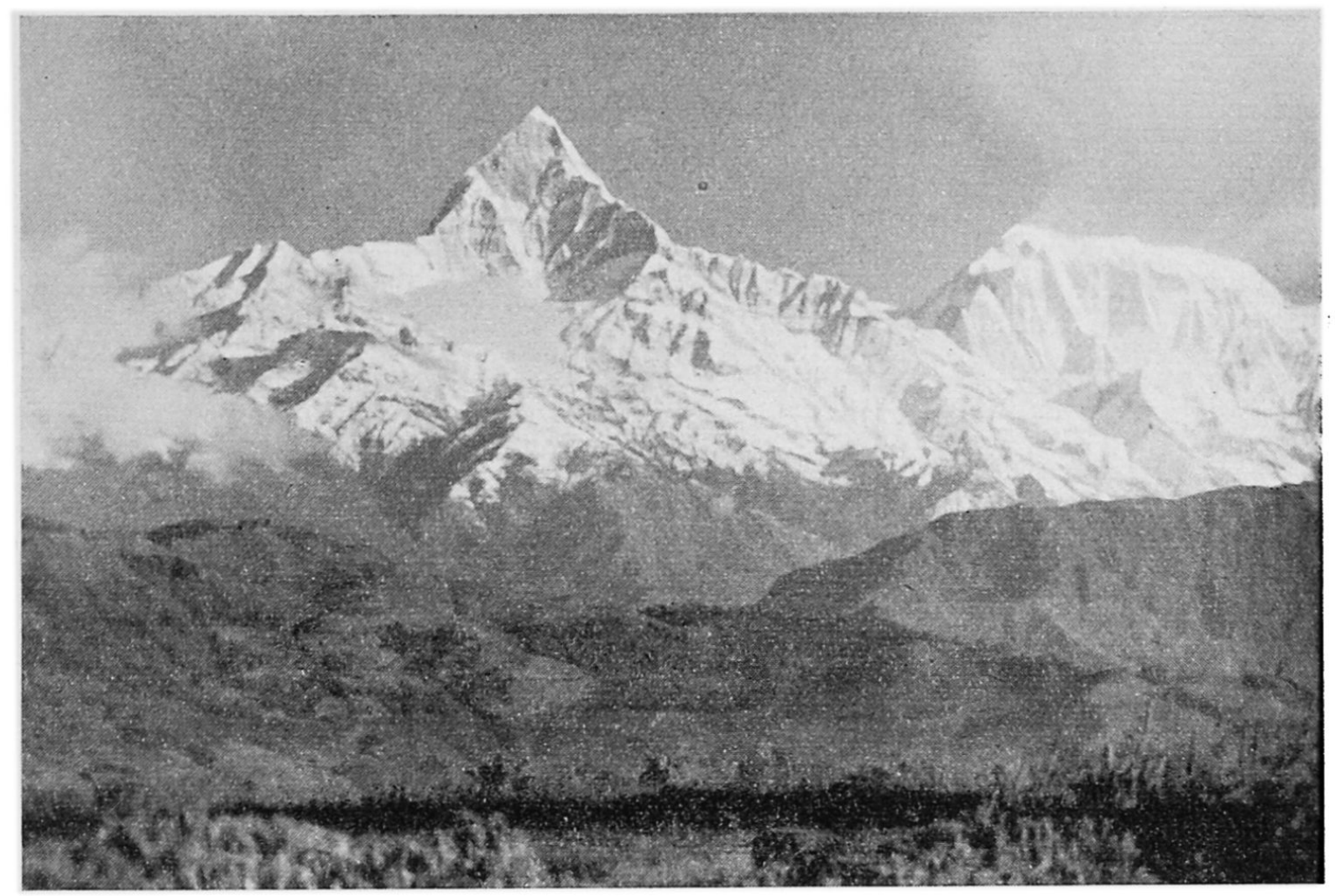

Vordergrund liegt rund $1000 \mathrm{~m}$ über Meer. Die Distanz zu den Gipfeln der Annapurna beträgt zu 7000 und $8000 \mathrm{~m}$. Von links nach rechts: Annapurna-Südgipfel (Gneise), Annapurna-HauptGipfelaufbau), Gangapurna (Basis Granite, Gipfel Kalke)

schon weiter nördlich, im Gebiet des heutigen Dana ist der Ur Kali ein zweites Mal angezapft worden, diesmal von einem westlichen Seitenfluß des Modi Kholas.

Es ist nicht erstaunlich, daß von der weiten Senke von Pokhara aus (1000 m ü. Meer) die Flüsse in Richtung der nun $50 \mathrm{~km}$ entfernten $8000 \mathrm{er}$ eine ganz gewaltige Erosionskraft entfaltet haben. Der Lauf des ehemaligen Ur-Kali wurde durch den Annapurna-Bogens nach Westen abgelenkt. Auch hier zeigt sich die zentrale Bedeutung des Annapurna-Bogens.

Wir wenden uns nun wieder dem Kali-Gandaki zu, der die Hauptkette zwischen Annapurna $(8078 \mathrm{~m}$ ) und Dhaulagiri $(8171 \mathrm{~m}$ ) (Luftlinie zwischen den Hauptgipfeln $30 \mathrm{~km}$ ) in der wohl gewaltigsten Schlucht der Erde (Talsohle bei Dana nur $1100 \mathrm{~m})$ durchbricht. Bei Lete $(2400 \mathrm{~m})$ öffnet sich der Talboden nach Norden wieder weit und erstreckt sich auf eine Länge von $35 \mathrm{~km}$ bis nach Chukhgaon hinauf. Ca. $600 \mathrm{~m}$ mächtige horizontal liegende Ablagerungen quartären Alters enthalten blaue Seetone und auch Salzlagerstätten. Das Salz von Tetang wird schon seit langem auf primitive Art abgebaut.

$15 \mathrm{~km}$ nördlich von Mustang befindet sich die Wasserscheide gegen den Tsangpo (Brahmaputra), also rund $80 \mathrm{~km}$ nördlich der Himalayakette. Es ist demnach gewi 3 , daß ehedem im Kali-Tal nördlich von Annapurna und Dhaulagiri ein See bestand. Die Salzlagerstätten deuten darauf hin, daß dieser See zeitweise abflußlos war. Als wichtige Beobachtung ist die beträchtliche Aufwölbung der gut geschichteten Quartärablagerungen gegen die Himalaya Hauptkette zu erwähnen (nördlich Kagbeni) (Fig. 3). Ein Vergleich all dieser Erscheinungen mit der Geologie zeigt, daß der Riegel, welcher den ehemaligen Stausee verursacht hat, genau mit den kristallinen Wurzeln der Kathmandu-Decken zusammenfällt

Es lassen sich daraus folgende Schlußfolgerungen ziehen: Das Tal der Gali Gandaki ist älter als die heutigen Formen der Hauptkette des Himalaya. Im Gebiet von 


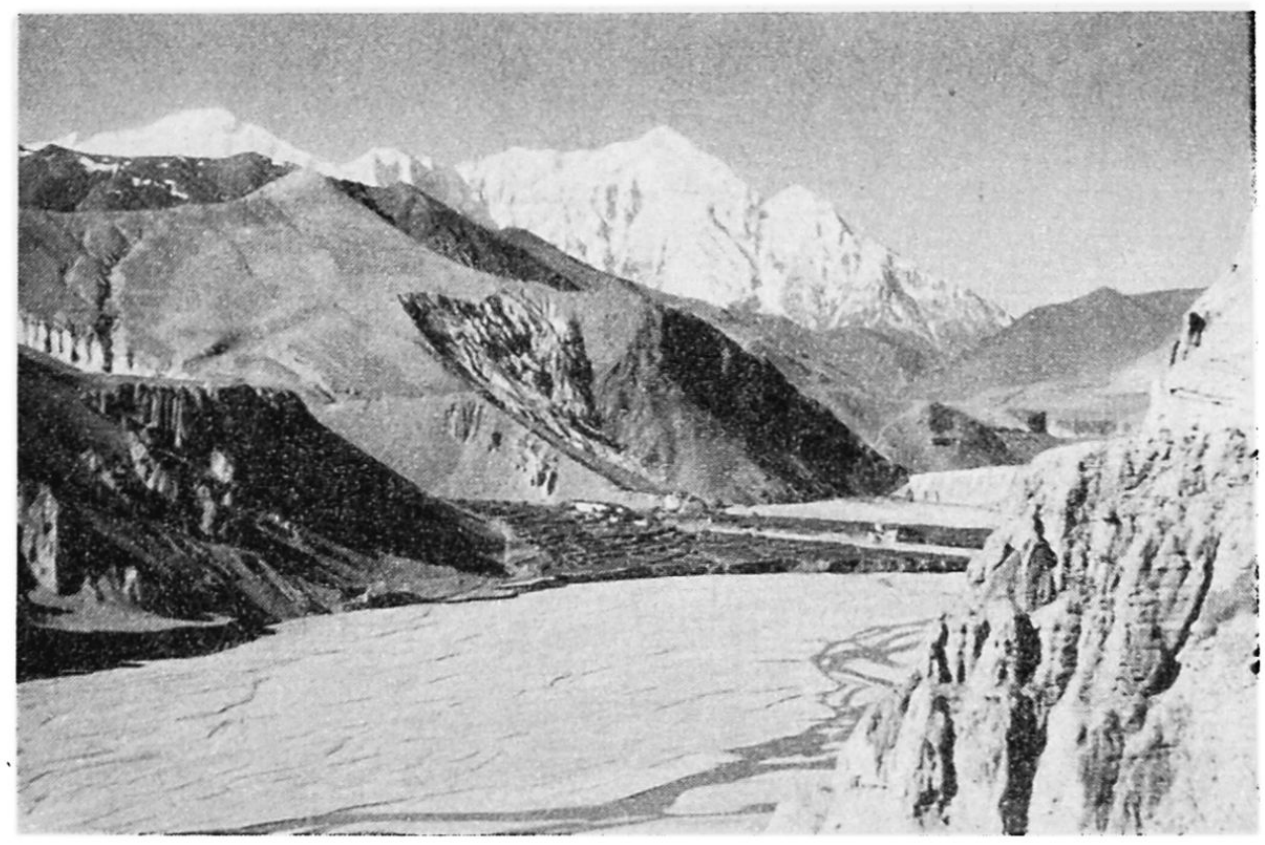

Fig.4. Im Kali-Tal, $2800 \mathrm{~m}$. Blick nach Süden gegen die Barriere der Annapurna.Ausgedehn. te quartäre Ablagerungen von meh. reren $100 \mathrm{~m}$ Mächtigkeit. Die Landschaft zeigt vollkommen Tibetischen Charakter. Das Klima ist trocken, und dementsprechend herrscht eine $\mathrm{Ge}$ birgswüste. Tibetische Siedlungen liegen auf künstlich bewässerten Schuttkegeln der Seitenbäche

Tukucha-Kagbeni war schon ein weiter Talkessel ausgeräumt, bevor die Hauptüberschiebung und Aufpressung der Wurzeln der Kathmandu-Decken erfolgte. Diese Emporpressung erfolgte so rasch, daß der Kali nicht Zeit hatte, gleichzeitig sein Bett entsprechend tiefer zu legen, ja, daß zeitweise in dem trockenen Klima nicht einmal mehr ein Abfluß nach Süden vorhanden war. Man kann also von einem tektonischen Stausee sprechen.

Eine ähnliche Erscheinung dürfte auch im Trisulital nördlich Kyerong Dzong vorliegen, sowie auch im Oberlauf des Langu Kholas.

Aber auch sämtliche andern großen und bereits genannten Durchbruchsflüsse zeigen analoge Längsprofile: Flachen Oberlauf in der tibetischen Zone und Randschuppenzone in Höhen bis ca. $2800 \mathrm{~m}$, Durchbruch durch die Himalaya-Hauptkette in den kristallinen Wurzeln der Kathmandu-Decken und wiederum flachen Unterlauf, wobei die tiefen Talböden von Süden her bis sehr nahe an die Hauptkette heranreichen (Pokhara $1000 \mathrm{~m}$; Tarpu am Marsyandi $900 \mathrm{~m}$; Setibus am Buri Gandaki $1400 \mathrm{~m}$; Syabrubensi am Trisuli $1700 \mathrm{~m}$ ).

Wie erwähnt, bilden die Wurzeln der Kathmandu-Decken die Hauptkette, und zwar besteht kein allmählicher topographischer Übergang von der Ganges Ebene über das Nepalische Mittelland zum Hochgebirge. Vielmehr besteht zwischen dem topfebenen Ganges-Alluvium und den Molasse-Ketten der Siwaliks (zwischen 600 und $1100 \mathrm{~m}$ ) eine scharfe Grenze. Nördlich an die Siwaliks schließt sich die Mahabharatkette $(2000-3000 \mathrm{~m})$ an, welche die südliche Brandungszone der Decken darstellt (ähnlich Säntis). Hernach folgt weiter nach Norden das dicht besiedelte Nepalische Mittelland mit Höhenlagen zwischen 800 und $2000 \mathrm{~m}$, und endlich, praktisch ohne Übergang, die Wandflucht des Hochgebirges. Besonders eindrücklich ist dies an der Annapurna etwa von Pokhara aus, oder auch am Dhaulagiri zu sehen (Fig. 4). Die Gruppe des letzteren bildet nach Süden eine einzige Steilwand von gegen $4000 \mathrm{~m}$ Höhe.

Die Betrachtung der überhöhten Querprofile (Fig. 5) zeigt sofort, daß die alles überragenden Hauptketten genau identisch mit den Wurzeln der Kathmandu-Decken sind. Das ganze Bewegungsbild in den geologischen Querprofilen deutet auf ein Emporpressen der Wurzelpartien in Folge horizontalen Schubes. Da die tibetische Platte bei diesem horizontalen Schub höher lag als das südliche Vorland, resultierte eine Überschiebung der Decken nach Süden. Bei diesem Emporpressen wurde aber auch 
Fig. 5. Die Nordfallenden Quartärformationen nördlich v. Annapurna und Dhaulagiri (bei Chukhgaon). Eine letzte Hebung derDeckenwurzeln in der Himalaya Hauptkette bewirkte das Aufbiegen der jungen Schichten gegen den Himalaya

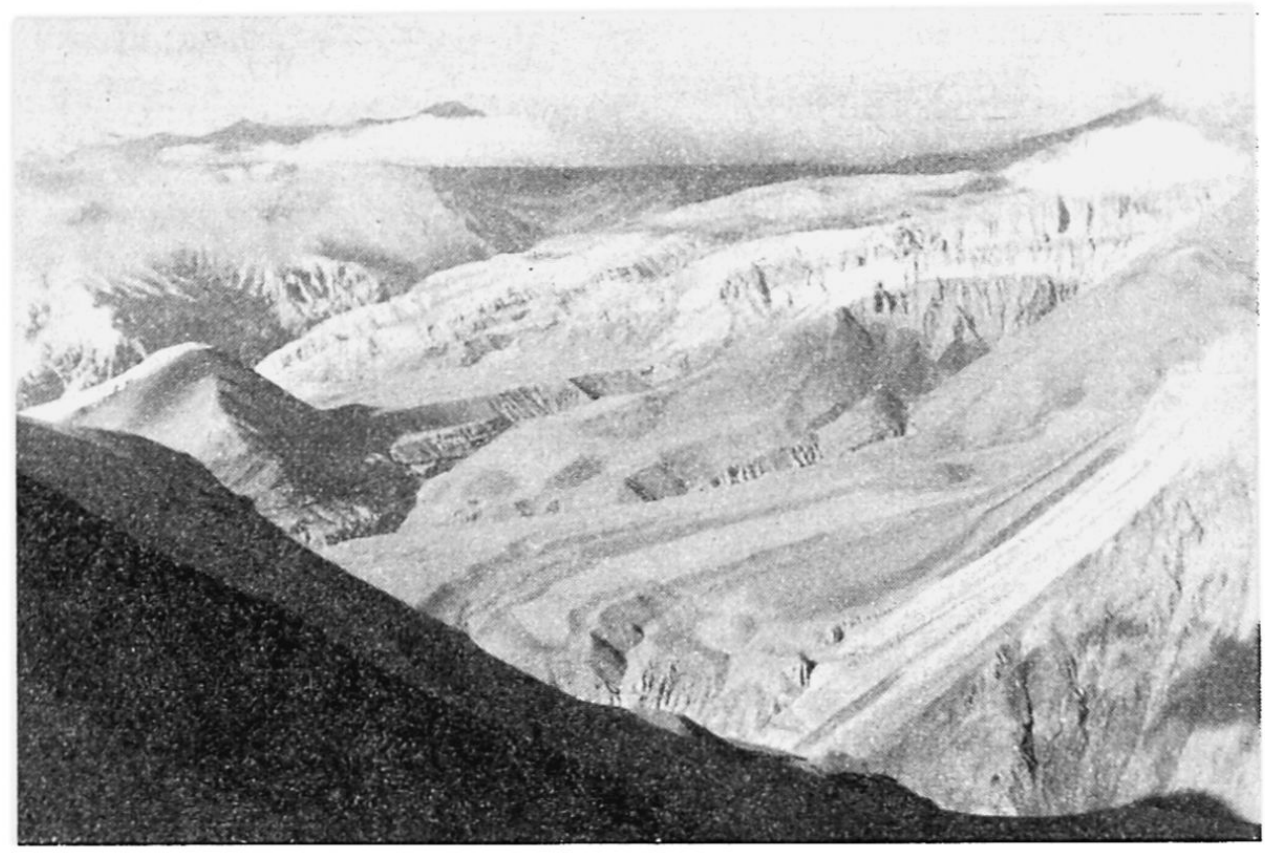

der Rand der Tibetischen Plateaus z. T. mitgenommen und gehoben, woraus wieder rückläufige (nach Norden gerichtete), Verschuppungen und Verfaltungen auftreten.

Heute liegen einige der höchsten Gipfel wie Annapurna, Manaslu im Kristallin der Kathmandu-Decken V, andere wie der Dhaulagiri etwas weiter nördlich, in der Sedimentbedeckung der Kathmandu-Decken V, der Ganesh-Himal und LangtangHimal in den Wurzeln der Kathmandu-Decken IV. Unter Berücksichtigung der Tatsache, daß die Erosion von Süden her bedeutend stärker wirkte als von Norden, lag die Flur der höchsten Gipfel gegen Ende der Haupthebung sicherlich etwas weiter südlich, in den Wurzeln der Kathmandu-Decken II-IV, also in der Mitte der steil gestellten Kathmandu-Decken Einheiten.

LOMBard (1953) hat im Everestgebiet 3 Kathmandu-Decken und 2 NuptseSchuppen ausgeschieden. Es ist nicht ausgeschlossen, daß die 2 Nuptse-Schuppen meinen Kathmandu-Decken IV und V entsprechen, mit der Kristallin Basis des Everest. Es ergibt sich auch hier wieder das gleiche Bild wie weiter im Westen, daß die höchste Erhebung in der nördlichsten Wurzeleinheit liegt.

Zusammenfassend kann festgestellt werden, daß es nicht nötig ist, die Isostasie in der oben erwähnten Form zur Erklärung der abnormen Höhe des Himalayagebirges heranzuziehen. Vielmehr entspricht die Hauptkette den Wurzeln der KathmanduDecken, die in einer relativ späten Phase horizontaler Zusammenschübe in die Höhe gepreßt worden sind. Es sind also auch im Himalaya aktive, horizontale Stoßkräfte als wesentlich für die Gebirgsbildung festzustellen, wie dies in den Alpen schon seit langem bekannt ist.

\section{LITERATUR}

Auden, J. B. (1935): Traverses in the Himalaya. Records Geol. Survey of India, Vol. 69/2; (1937): The structure of the Himalaya in Garhwal. Records Geol. Survey of India, Vol. 71. BuRRard, S. G. und HAYDEN, H. H. (1934): A sketch of the geography and geology of the Himalaya Mountains and Tibet, Delhi. Garwood, E. J. (1903) in D. W.: Freshfields „Round Kangchendzönga“, London. Hagen, T. (1951): Preliminary note on the Geological structure of Central Nepal. Verhdlg. der Schweiz. Naturforschenden Gesellschaft, Luzern; (1952): Flug eines Geologen über Nepal. Atlantis, Märzheft 1952. Hein, A. und Gansser, A. (1938): Geological Observations in the Central Himalaya. Denkschriften der Schweiz. Naturforschenden Gesellschaft, Bd. 58. Lombard, A. (1953): Les racines des nappes de Kathmandu dans le Népal oriental et les nappes de Khumbu. Archives des sciences, société de physique et d'histoire naturelle de Genève. MidDi.Emiss, C. S. (1891): Mem. Geol. Survey of India, Vol 24. NANsen, F. (1928): The Earth's crust, its surface forms and isostatic adjustement. Norske Videnskaps-Akademi, Nr. 12, Oslo. Oder.L (1925): Geogr. Journal 66. De Terra, H. (1934): Geographical Review, Vol. 24. Wager, L. R. (1937): The Arun River drainage pattern and the rise of the Himalaya. The Geographical Journal, Vol. no 3. 
The remarkable behaviour of many rivers in cutting across from the Tibetan plateau through the much higher Himalayan range has given rise to two main theories. One of them postulates that at an early stage the Himalaya had ordinary, consequent drainage, and that in a later stage south-flowing rivers were cutting back through the range capturing rivers on the Tibetan side. The alternative theory postulates that the rivers had their present courses before the Himalayan range had risen up across the rivers and that the latest upwarping of the Himalayas was caused by isostatic reasons due to the effect of loading and unloading the crust of the earth by the Quaternaty ice sheet and (or) by erosion of the deep valleys at the southern edge of the Tibetan plateau.

The author found that the main range of the Himalaya corresponds with the roots of the large nappes (Kathmandu nappes). The roots were exactly raised up so rapidly in a late stage to the present height, that the rivers flowing south from far north long before, had not time to cut their riverbeds in the uprising Himalayas correspondingly. In the valley of the Kali Gandaki a large tectonic lake was formed by the dam of the rising Himalayas. The lake is proved by lake deposits and salt formations. These quaternary formations show a remarkable northern dip of about $18^{0}$ near Tetang (north of Annapurna). All the big rivers cross the main range between different tectonic arcs, each group of the high mountains forming such an arc.

\section{LA GEOGRAPHIE A LA $134^{\text {me }}$ SESSION ANNUELLE DE LA SOCIETE HELVETIQUE DES SCIENCES NATURELLES}

\section{Willy DeRron}

Selon la décision du Comité central et du Sénat de la S. H. S. N., l'assemblée annuelle de 1954 avait lieu à Altdorf, du 25 au 27 septembre. Le comité local n'a pu assumer sạ tâche qu'au prix d'une simplification considérable de l'organisation matérielle: ses moyens limités ont été consacrés à la science, ce qui l'a contraint à renoncer à tout apparat. Mais on trouvait à Altorf la chaude et traditionnelle hospitalité uranaise, et c'était un esprit de camaraderie qui animait les réunions scientifiques, les discussions, le travail en commun.

A l'occasion de la réunion annuelle de la S.H.S.N., notre Fédération a tenu une séance où étaient représentées la plupart de nos sociétés. L'ordre du jour, présenté par le vice-président du Comité central, M. Linıger, prévoyait la question de la réorganisation de l'Union internationale des sociétés scientifiques. A la majorité des voix, il fut décidé d'écarter la proposition de M. de Muralt et de s'en tenir à l'ordre actuel. Puis il s'agissait de désigner un représentant de la Fédération dans un comité consultatif pour la publication d'un glossaire géographique international. L'idée émane du professeur E. KaNT, de l'université de Lund, à qui l'assemblée propose, pour tenir compte de notre bigarrure linguistique, les noms de MM. WIDMER, St-Gall, et PerRET, Genève. M. le prof. DAMI sera prié de prêter son précieux concours, pour ce qui touche à l'italien.

Après l'assemblée, six conférenciers tinrent leur auditoire sous le charme de leurs très originales présentations. Comme l'a fort bien relevé le vice-président, ces communications étaient bien faites pour donner une idée favorable de la vaste activité de notre Fédération, qui embrasse tous les domaines, si variés et si étendus, de la géographie.

Suivent les résumés individuels des communications:

Hagen, Tonı, Kathmandu: Über Gebirgsbildung und Talsysteme im Nepal-Himalaya, paru in extenso dans Geographica, p. $325-332$.

Carol, Hans, Zürich: Landschaftskundliche Forschungen in Ostafrika. Im Rahmen meines dreimonatlichen Aufenthaltes verwendete ich einen Monat für ausgedehnte Reisen, um Ostafrika überblicksnäßig kennen zu lernen und zwei Monate für die Untersuchung von zwei kleinen, aber typischen Ausschnitten der ostafrikanischen Landschaft. Schon zu Hause wählte ich diese zwei, in allen Beziehungen extremen Landschaften aus: ein extensives Weidegebiet in den trockenen Buschsavannen Nordost-Ugandas und ein feuchtes, intensiv bewirtschaftetes Ackerbaugebiet im Bananengürtel westlich Kampala. Diese Landschaften wurden vom agrargeographischen Gesichtspunkte aus, d. h. unter dem Aspekt der landwirtschaftlichen Nutzung untersucht. 